\title{
Ostéopétrose ou maladie des os de marbre 2. Pathogénie et traitement
}

\section{Osteopetrosis or marble bone disease 2. Pathogeny and treatment}

YVES GERMANIER, JEAN-PIERRE CARREL, SEMAAN ABI NAJM, JACKY SAMSON

\section{RÉSUMÉ}

Le remodelage osseux joue un rôle important dans la formation, la croissance, l'adaptation, la conservation et la réparation du squelette. Ce remodelage s'effectue sous l'action coordonnée des ostéoblastes et ostéoclastes. L'activité des ostéoclastes dépend de celle des ostéoblastes mais aussi de bien d'autres facteurs. L'expérimentation animale a déjà permis d'identifier plusieurs d'entre eux.

Dans l'ostéopétrose, l'activité des ostéoclastes est perturbée et on dispose de peu de moyens pour leur redonner une fonction normale. Dans l'ostéopétrose maligne, seul l'apport de nouveaux ostéoclastes par transplantation de moelle osseuse permet de stopper ou de ralentir l'évolution. (Med Buccale Chir Buccale 2006; 12: 149-57).

mots clés : ostéopétrose, ostéoclastes, remodelage osseux, transplantation de moelle osseuse

\section{SUMMARY}

Bone remodelling play an important role in the formation, growing, adaptation, conservation and reparation of the skeleton. This remodelling is performed by coordination of osteoblasts and osteoclasts. Osteoclast function depends on the osteoblast activity and from many others factors.

Animal experimental studies have already identified several of these factors. In osteopetrosis, there is a dysfunction of osteoclast and we don't have so many ways to restore there function. Transplantation of bone marrow in malignant osteopetrosis can slow down or stop the evolution of the disease by the contribution of new osteoclasts.

(Med Buccale Chir Buccale 2006; 12: 149-57).

key words : osteopetrosis, osteoclasts, bone remodelling, bone marrow transplantation 


\section{PATHOGÉNIE}

\subsection{Généralités}

L'os est un tissu conjonctif spécialisé qui, avec le cartilage, compose le squelette. Ce dernier a de multiples fonctions où l'on discerne trois rôles principaux : (i) mécanique : par leur rigidité et leur dureté, les os permettent au squelette de maintenir la forme générale du corps et servent de point d'attache aux muscles pour la locomotion ; (ii) protecteur : en protégeant les organes vitaux contenus dans la boîte crânienne et la cage thoracique, et la moelle osseuse hématopoïétique ; (iii) métabolique : en servant de réserve ionique pour l'organisme entier, principalement pour le calcium et les phosphates dont l'homéostase est indispensable à la vie ${ }^{[85,86]}$.

D'un point de vue anatomique, on distingue classiquement deux types d'os dans le squelette : les médecine buccale chirurgie buccale

VOL. $12, \mathrm{~N}^{\circ} 3$ 2006

page 150 os plats (os du crâne, omoplate, mandibule, bassin) et les os longs (tibia, fémur, humérus...), ayant respectivement une ossification membraneuse et enchondrale, bien que la croissance et le développement ultérieurs des os longs fassent appel aux deux phénomènes ${ }^{[5,86]}$. L'os long comporte une partie cylindrique centrale, la diaphyse, aux deux extrémités de laquelle on trouve des structures arrondies, les épiphyses. La région en forme d'entonnoir qui relie la diaphyse à chaque épiphyse constitue la métaphyse. Alors que la diaphyse est essentiellement composée d'os compact (ou cortical), qui délimite la cavité médullaire contenant la moelle hématopoïétique, les épiphyses et les métaphyses sont constituées d'os spongieux (ou trabéculaire), recouvert d'une

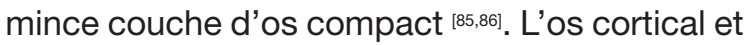
l'os trabéculaire sont constitués par les mêmes cellules et la même matrice, mais présentent des différences structurelles et fonctionnelles. Sur le plan structurel, on constate que $80-90 \%$ du volume de l'os compact est calcifié alors que cette proportion est de 15 à $25 \%$ dans l'os spongieux, le volume restant étant occupé par la moelle osseuse, les vaisseaux sanguins et le tissu conjonctif. Leurs différences fonctionnelles sont liées à la structure : l'os cortical remplit principalement un rôle mécanique et protecteur, l'os spongieux assure plutôt un rôle métabolique ${ }^{[85,86]}$.
L'élaboration du tissu osseux comprend de nombreuses étapes comportant successivement la synthèse d'une matrice primaire secondairement minéralisée, l'adjonction de matrice là où l'exigent des vecteurs de force et de croissance, et la résorption de l'os en excès. Dans les cartilages de conjugaison, on observe une calcification associée à une résorption partielle du cartilage hyalin déposé par les chondroblastes après qu'il ait servi à l'élaboration de l'os fibreux. Au cours des phénomènes de croissance et de remodelage ultérieurs, les travées osseuses primitives sont progressivement résorbées par les ostéoclastes et de nouvelles travées osseuses composées d'os lamellaire sont formées, permettant ainsi l'élaboration d'une architecture osseuse adaptée aux contraintes mécaniques avec une limite cortico-médullaire nette ${ }^{[85,86]}$. Dans l'ostéopétrose, le manque de résorption osseuse empêche le déroulement harmonieux des différentes étapes fondamentales de l'ossification enchondrale. L'os fibreux ainsi que le cartilage hyalin calcifié persistent in situ et interfèrent avec la mise en place de l'architecture osseuse normale, et avec le développement des cavités médullaires et de la moelle hématopoïétique [24,79,87]. L'os qui en résulte, bien que plus dense, se caractérise par une résistance mécanique nettement

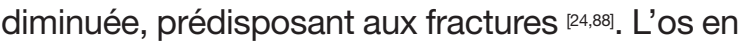
excès entraîne progressivement la diminution du volume des cavités médullaires avec étouffement de la moelle osseuse et la compression des pédicules vasculaires nourriciers, ce qui rend l'os particulièrement vulnérable face à l'infection [3,89-91]

\subsection{Mécanismes cellulaires respon- sables de la résorption osseuse}

Les ostéoclastes sont des cellules géantes (20$100 \mu \mathrm{m})$, multinucléées (2-50 noyaux), provenant de la fusion dans le tissu osseux de précurseurs sanguins communs aux macrophages, aux cellules dendritiques et aux ostéoclastes [86]. A l'issue de la phase initiale de différenciation, les précurseurs myélomonocytaires peuvent s'engager dans différentes voies pour donner des macrophages, des cellules dendritiques ou des ostéo-

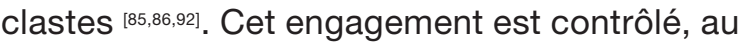
moins en partie, par c-Fos, facteur de transcrip- 
tion appartenant au complexe AP1, qui favoriserait la voie ostéoclastique par rapport aux voies macrophagique et dendritique ${ }^{[86,92]}$. En effet, on constate expérimentalement que les souris présentant une invalidation du gène c-Fos $\left(\mathrm{C}-\mathrm{Fos}^{-/-}\right)$, possèdent davantage de macrophages que les

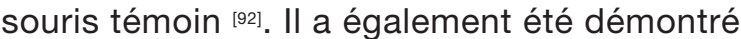
in vitro que c-Fos active la différenciation des ostéoclastes et inhibe celle des cellules dendritiques ${ }^{192]}$. Deux autres facteurs sont essentiels à cette première étape de l'ostéoclastogenèse : le M-CSF (Macrophage-Colony Stimulating Factor, également appelé CSF-1) et le RANK-L (Receptor Activator of Nuclear factor $\mathrm{\kappa B}$-Ligand) également appelé ODF (Osteoclast Differenciation Factor), OPGL (Osteoprotegerin Ligand) ou TRANCE (TNF-Related Activation-Induced Cytokine) ${ }^{[86,92] .}$ Ces facteurs sont synthétisés par les cellules du microenvironnement osseux et les ostéoblastes, et ils se fixent sur leurs récepteurs respectifs cFMS (également appelé récepteur du CSF-1) et RANK (Receptor Activator of Nuclear factor $\kappa B$ ), à la surface des pré-ostéoclastes ${ }^{[86,92]}$. Ceux-ci fusionnent pour donner des ostéoclastes multinucléés qui se polarisent au contact de l'os où ils sont activés [92]. L'ostéoprotégérine (OPG) agit comme régulateur de l'ostéoclastogénèse en se fixant à RANK-L et en bloquant son effet sur RANK : elle inhibe donc la différenciation des précurseurs ostéoclastiques ${ }^{\text {[2] }}$ (Fig. 1).

Les ostéoclastes activés siègent dans des dépressions de la surface osseuse, appelées lacunes de Howship, qui résultent de leur activité résorbante ${ }^{[86]}$. Au cours du processus de résorption osseuse, l'ostéoclaste adhère à l'os au moyen d'une structure spécialisée appelée zone claire. Celle-ci est constituée par une partie de la membrane cellulaire, dépourvue d'organelles et riche en filaments d'actine, qui s'applique de manière intime sur la surface osseuse [93-96]. Elle délimite ainsi une zone imperméable aux ions qui isole du milieu extracellulaire une autre partie de la membrane de l'ostéoclaste comportant une bordure en brosse, appelée lacune de résorption. Dans cette zone où le $\mathrm{pH}$ est acide, l'activité extracellulaire des enzymes lysosomiales induit la dégradation enzymatique du tissu osseux [86]. L'attachement de l'ostéoclaste à la surface osseuse est assurée par des intégrines qui se fixent à des protéines de la matrice osseuse comme le collagène, la fibronectine et la vitronectine ${ }^{[86]}$. La Fig. 2 illustre, de façon schématique, les différents mécanismes responsables de la résorption osseuse. Elle s'effectue sous le contrôle de nombreuses hormones et cytokines. Les facteurs inhibiteurs (calcitonine, prostaglandines ou CSF-1) agissent directement sur l'ostéoclaste en se fixant sur des récepteurs spécifiques disposés à sa surface [96-100]. En revanche, les facteurs favorisants $(\mathrm{PTH}$, calcitriol, IL1 ou TNF) interviennent de manière indirecte en stimulant la libération de cytokines par les ostéoblastes ${ }^{[96-98,100]}$. Ces derniers, d'origine mésenchymateuse, facilitent également la résorption osseuse en dégradant, par l'intermédiaire de collagénases et d'activateurs du plasminogène, la matrice protéique qui recouvre la surface osseuse et qui empêche l'attachement des ostéoclastes [96]. Le phénomène de résorption osseuse nécessite l'interaction de deux types distincts de cellules. L'un, d'origine hématopoiétique (l'ostéoclaste et ses précurseurs), est directement impliqué dans

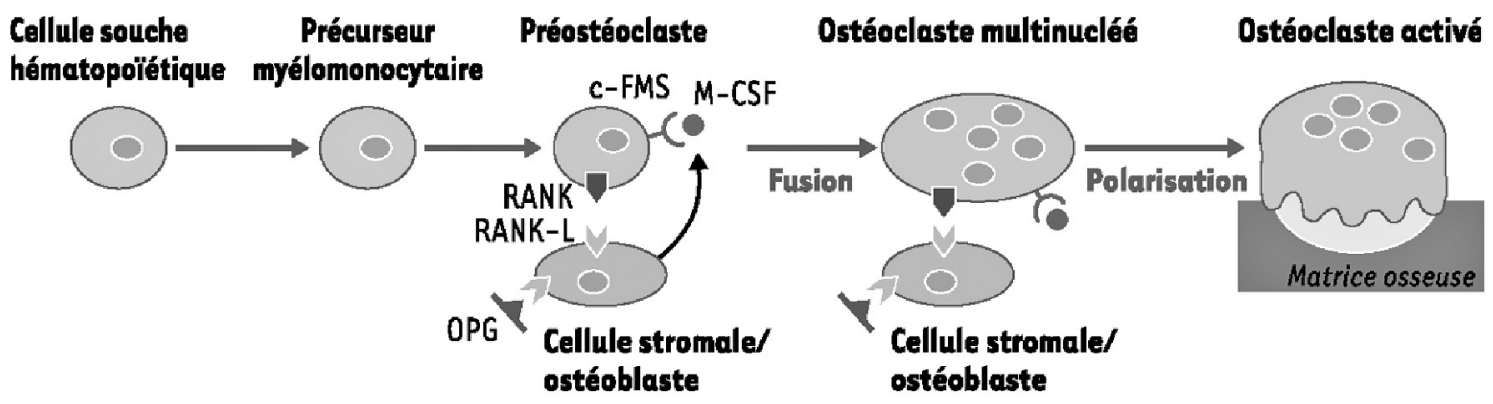

Figure 1 : Différentes étapes de la différenciation des ostéoclastes [92]. Different stages of osteoclast differenciation ${ }^{\left[{ }^{[2]}\right]}$. 
médecine

buccale

chirurgie

buccale

VOL. $12, \mathrm{~N}^{\circ} 3$ 2006

page 152 le processus de résorption osseuse. L'autre, d'origine mésenchymateuse (l'ostéoblaste), crée le micro-environnement favorisant le développement et l'activation de l'ostéoclaste. Tout processus qui interfère soit dans la différenciation soit dans l'activité des ostéoclastes, peut potentiellement être à l'origine d'un trouble de la résorption osseuse, donc d'une ostéopétrose [96]. Les données physiopathologiques permettent de distinguer schématiquement deux types d'ostéopétrose : l'une comportant des altérations dans la lignée cellulaire d'origine hématopoïétique et l'autre dans la lignée mésenchymateuse. Les cellules précurseurs hématopoïétiques pouvant être transplantées d'un donneur à un receveur, toute ostéopétrose causée par un défaut intrinsèque de cette lignée cellulaire est, en théorie, susceptible d'être traitée par une transplantation de moelle osseuse. A partir de 1972 le traitement de certaines formes d'ostéopétrose a connu une importante évolution avec les travaux de Walker : il démontra que l'on pouvait guérir des souches de souris ostéopétrotiques en les mettant en parabiose avec des souris saines et que la transplantation de moelle osseuse permettait de corriger le déficit de la résorption osseuse [101,102]. Ces travaux fournirent non seulement une nouvelle approche dans le traitement de l'ostéopétrose humaine par le biais de la transplantation de moelle osseuse, mais démontrèrent également le rôle joué par les monocytes circulants comme précurseurs des ostéoclastes [103-105].

\subsection{Principaux mécanismes pathogé- niques de l'ostéopétrose commune}

Les ostéoclastes sont des éléments essentiels pour le remodelage osseux et les anomalies de leur différenciation ou de leur activité conduisent à un trouble de la résorption osseuse responsable d'un phénotype ostéopétrotique. En théorie, les causes potentielles d'ostéopétrose sont nom-

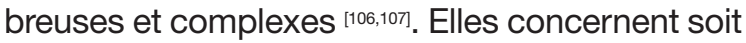

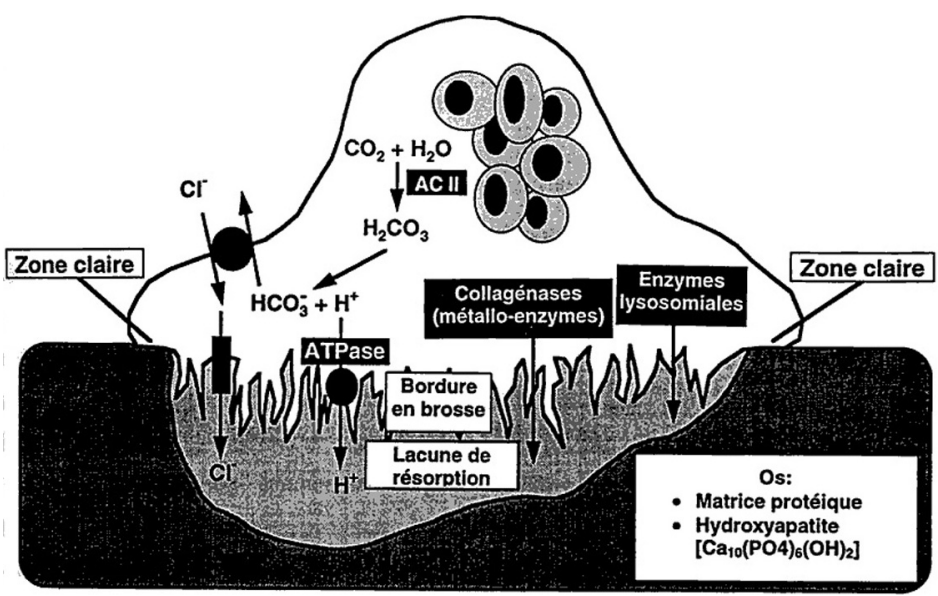

Figure 2 : Différents mécanismes intervenant dans la résorption osseuse par les ostéoclastes. Les ostéoclastes expriment à leur surface de nombreuses enzymes et protéines de transport. L'anhydrase carbonique II (AC II) catalyse la synthèse de l'acide carbonique $\left(\mathrm{H}_{2} \mathrm{CO}_{3}\right)$ à partir de $\mathrm{CO}_{2}$ et d' $\mathrm{H}_{2} \mathrm{O}$. Les protons, issus de la dissociation spontanée de l'acide carbonique, sont transportés dans la lacune de résorption par une pompe de la bordure en brosse. Un antiport échange le bicarbonate resté dans le cytoplasme contre du $\mathrm{Cl}^{-}$extra-cellulaire, qui va rejoindre les protons dans la lacune de résorption. L'acide chlorhydrique ainsi formé dissout les cristaux d'hydroxyapatite, rendant la matrice organique de l'os accessible à la dégradation enzymatique (d'après Felix et coll.) [96].

Different mechanisms involved in bone resorbtion by osteoclasts. Many transport enzymes and proteins are expressed on osteoclasts surface. Carbonic anhydrase II (CA II) catalyse the synthesis of carbonic acid $\left(\mathrm{H}_{2} \mathrm{CO}_{3}\right)$ from $\mathrm{CO}_{2}$ and $\mathrm{H}_{2} \mathrm{O}$. The protons, deriving from the spontaneous dissociation of carbonic acid, are transported in the pool of resorption through a pomp at the ruffled border. An antiport exchange the bicarbonate staying in the cytoplasm against extracellular $\mathrm{Cl}^{\prime}$ and the $\mathrm{Cl}^{\prime}$ will rejoin the protons in the pool resorption. The formed chlorydric acid will dissolve the hydroxyapatite crystals which lead the enzymatic degradation of the organic matrice possible (from Felix and al.) ${ }^{[96]}$. 
les cellules précurseurs des ostéoclastes, soit les cellules mésenchymateuses qui constituent le micro-environnement des ostéoclastes ou les facteurs de croissance favorisant leur différenciation et leur maturation ${ }^{[3,24,108]}$. Les ostéoclastes peuvent présenter des anomalies lysosomiales responsables d'un défaut de la résorption osseuse, une résistance à certaines hormones ou un trouble de reconnaissance de l'os qui doit être résorbé, la matrice squelettique des altérations de structure la rendant réfractaire à la dégradation ostéoclas-

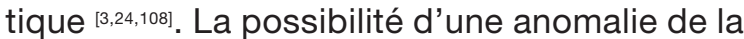
fonction des ostéoclastes a été confirmée en 1996 chez deux patients atteints d'ostéopétrose maligne ${ }^{[24]}$. Dans l'ostéopétrose bénigne, plusieurs travaux évoquent une probable résistance des ostéoclastes aux hormones thyroïdiennes et à la vitamine $1,25(\mathrm{OH})_{2} \mathrm{D}_{3}$, respectivement dans les types I et II [38,10].

D'autres anomalies sont fréquemment évoquées dans la pathogenèse de l'ostéopétrose. Une altération intrinsèque de la fonction phagocytaire des granulocytes et des monocytes, généralement observée dans la forme maligne, pourrait expliquer l'association d'un trouble de la résorption osseuse et d'une prédisposition accrue aux infec-

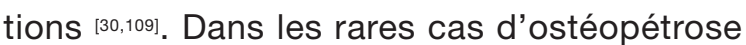
associés à une maladie de stockage neuronal, plusieurs études anatomopathologiques ont révélé des altérations dégénératives touchant les neurones, caractérisées par une dystrophie axonale généralisée, la présence de corps sphéroïdes, et par l'accumulation de céroïdo-lipofuschine. Ces observations suggèrent qu'une altération de la fonction lysosomiale pourrait être la cause de cette forme d'ostéopétrose ${ }^{[15] .}$. Certains cas d'ostéopétrose pourraient être dus à un déficit de production de I'IL-2 [111], une synthèse de la PTH anormale ${ }^{[112]}$ ou une production insuffisante de facteurs nécessaires à la résorption osseuse comme les superoxydes ${ }^{[24]}$. En effet, il est parfois possible d'induire une stimulation de la résorption osseuse avec le CSF-1, l'IL-2 ou l'IFN- $\gamma$, ou en augmentant la synthèse des superoxydes par les granulocytes et les monocytes [113-115]. Une étude récente a montré l'efficacité de l'IFN- $\gamma$ dans le traitement de l'ostéopétrose maligne chez l'enfant ${ }^{[15]}$. Enfin, certaines formes d'ostéopétrose semblent avoir une origine infectieuse : plusieurs études réalisées en microscopie électronique suggèrent que des virus comme le paramyxovirus, le virus respiratoire syncytial ou celui de la rougeole retrouvés dans les ostéoclastes, pourraient être responsables de formes sporadiques d'ostéopétrose bénigne ${ }^{[18]}$.

\subsection{Anomalies génétiques dans l'os- téopétrose humaine}

Les formes héréditaires de l'ostéopétrose humaine semblent toutes se transmettre selon un mode autosomal, dominant ou récessif, et ont toutes comme dénominateur commun un trouble de la résorption osseuse lié aux ostéoclastes. Différents modèles murins développant une ostéopétrose secondaire à la mutation spontanée ou à l'invalidation de gènes ont permis d'élucider en partie les mécanismes impliqués dans la différenciation et l'activité des ostéoclastes [92]. Trois modèles murins, les souris $\mathrm{C} / \mathrm{CN} 7^{-/}$, oc/oC et $g / / g l$ présentent un phénotype proche de celui de l'ostéopétrose maligne infantile ; ils constituent donc des modèles particulièrement adaptés à l'étude de l'ostéopétrose humaine [3,22]. Des mutations dans les gènes CICN7, TCIGR1 et GL ont donc été recherchés chez des patients atteints d'ostéopétrose : elles ont été retrouvées surtout dans l'ostéopétrose maligne infantile et parfois dans l'ostéopétrose bénigne de type || [3,92].

La dissolution de la matrice minérale nécessite une acidification de la lacune de résorption afin que les enzymes protéolytiques participant à la dégradation de la matrice osseuse organique aient une activité optimale ${ }^{[86]}$. Elle fait intervenir la pompe $\mathrm{H}^{+}$-ATPase vacuolaire et le canal chlore boy (Fig. 2). Ce système d'acidification est altéré dans deux modèles d'ostéopétrose murine $c / c n 7^{-/-}$et oc/oc [3,92]. Des mutations du gène CICN7 situé sur le chromosome 16p13.3 ont également été recherchées chez l'homme et détectées principalement dans l'ostéopétrose bénigne de type II, et plus rarement dans la forme maligne [92]. Dans ces mutations, on observe des insertions, délétions ou mutations non-sens et faux-sens touchant les acides aminés conservés chez tous les membres de la famille des protéines du canal chlore $\mathrm{CIC} 7{ }^{\text {[92]. L'invalidation du gène }}$ médecine

buccale

chirurgie

buccale

VOL. $12, \mathrm{~N}^{\circ} 3$ 2006

page 153 
médecine

buccale

chirurgie

buccale

VOL. $12, \mathrm{~N}^{\circ} 3$ 2006

page 154
CICN7 induit une altération du canal chlore CIC7 responsable du phénotype ostéopétrotique. Cependant, des patients porteurs de mutations du gène CICN7 peuvent avoir un phénotype tantôt sévère comme dans l'ostéopétrose maligne infantile, tantôt bénin comme dans l'ostéopétrose adulte, pour des raisons qui restent encore à élucider.

L'autre élément essentiel du système d'acidification de la lacune de résorption fait intervenir la pompe $\mathrm{H}^{+}$-ATPase vacuolaire (Fig. 2). Des altérations de cette pompe sont retrouvées chez les souris oc/oc ${ }^{192]}$ qui présente une mutation spontanée du gène TCIRG1 ( $T$ cell immune regulator 1 ), également appelé ATP6i, codant pour l'isoforme $\alpha 3$ de la sous-unité de $116 \mathrm{kDa}$ de la V-ATPase ${ }^{[22]}$. Des mutations du gène TCIRG1 ont été retrouvées dans environ $50 \%$ des cas d'ostéopétrose maligne [24,92]. Ces mutations entraînent probablement la synthèse de protéines non fonctionnelles, d'ARNm instable ou ne pouvant pas être traduit correctement ${ }^{[2]}$. Cependant, toutes ces mutations ne sont pas présentes à l'état homozygote : certains patients sont des hétérozygotes composites et, dans certains cas, les mutations ne sont détectées que sur un seul allèle du gène ${ }^{[02]}$.

Une étude récente, portant sur 19 patients atteints d'ostéopétrose maligne, qui ne présentaient pas de mutations dans les gènes TCIRG1 et CICN7, retrouve une mutation du gène GL chez un seul patient ${ }^{[22]}$. Les souris portant la mutation $\mathrm{gl} / \mathrm{gl}$ développent une anomalie de la pigmentation et une ostéopétrose sévère caractérisée par une altération du cytosquelette et de la formation de la bordure en brosse des ostéoclastes. Le gène gl muté chez ces souris code pour une protéine cytoplasmique dont le rôle n'a pas encore été élucidé ${ }^{[2]}$.

Les souris $\mathrm{ClCN} 7^{-/}$, oc/oc et $\mathrm{gl} / \mathrm{gl}$ constituent des modèles prometteurs pour l'étude de l'ostéopétrose, car elles représentent le même phénotype que celui de l'ostéopétrose maligne infantile, et les bases génétiques de cette ostéopétrose sont les mêmes que celle observées dans la forme humaine.

A la fin des années 90 , le rôle primordial, dans l'ostéoclastogénèse, du ligand de RANK (RANK-
L), appartenant à la superfamille du TNF (Tumor Necrosis Factor), de son récepteur RANK (Receptor Activator of Nuclear Factor $\kappa B$ ) et de l'ostéoprotégérine a été mis en évidence ${ }^{[2]}$. La liaison de RANK-L avec RANK déclenche une cascade de signalisation impliquant, en particulier, l'activation du facteur de transcription dimérique NF- $\mathrm{KB}{ }^{[02]}$. Chez la souris, l'invalidation des gènes impliqués dans la synthèse du facteur de transcription NF- $\kappa B$ entraîne un dysfonctionne-

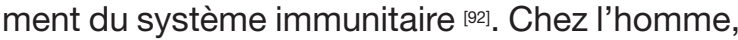
le syndrome OL-EDA-ID associant ostéopétrose, lymphoedème, dysplasie ectodermique anhydrotique et immunodéficience ${ }^{[23]}$ est précisément causé par une mutation du gène codant le facteur de transcription NF-KB ${ }^{[24]}$.

\subsection{Mécanismes pathogéniques dans l'ostéopétrose due à un déficit en AC II}

La description en 1983 d'un syndrome associant ostéopétrose, acidose tubulaire rénale et calcifications cérébrales confirme le rôle physiologique majeur joué par cette enzyme dans le rein, le cerveau, les os et les globules rouges [3,10]. Parmi les 4 types d'AC et les isoenzymes apparentées (carbonate-déhydratase, carbonate-hydrolyase) connus chez les mammifères, c'est l'AC II qui possède la plus large distribution tissulaire, la plus grande activité catalytique et le turn-over le plus rapide $[24,63,116]$.

L'activité des AC est relativement intense dans les ostéoclastes, plus particulièrement celle de I'AC II qui peut être stimulée par la PTH ${ }^{[49,117-119] . ~ L e ~}$ déficit en AC II dans les ostéoclastes semble bloquer la transformation du $\mathrm{CO}_{2}$ et de $\mathrm{H}_{2} \mathrm{O}$ en $\mathrm{H}_{2} \mathrm{CO}_{3}$, donc le transport de $\mathrm{H}^{+}$et l'acidification du milieu extracellulaire. De fait, l'inhibition pharmacologique de l'AC, par exemple par l'acétazolamide, entraîne in vitro et in vivo un arrêt de la résorption osseuse ${ }^{[120,121]}$.

L'origine des calcifications cérébrales reste inconnue. L'AC II constitue la principale isoenzyme de la famille des $\mathrm{AC}$ dans le cerveau et son pic d'activité semble se situer vers l'âge de trois ans-etdemi [122], âge qui correspond à celui de la découverte radiologique de premières calcifications cérébrales chez les enfants atteints d'un déficit en AC || [73]. La PTH peut augmenter le taux 
d'AC dans les cellules osseuses en culture [123,124] et les calcifications liées au déficit en AC II sont comparables à celles que l'on observe couramment dans l'hypoparathyroïdie idiopathique ou la pseudohypoparathyroïdie [54].

Paradoxalement, les patients ayant un déficit en AC II ne présentent pas d'altération de leurs sécrétions salivaires, gastriques ou pancréatiques : la présence des autres isoenzymes (AC I, III ou IV) est probablement suffisante pour assurer ces sécrétions. Dans le déficit en AC II, l'étude des globules rouges montre que l'activité de l'AC I apparaît suffisante pour permettre la réalisation des des échanges gazeux sanguins [63].

Le gène de l'AC II a été découvert chez l'homme en 1991 : il est situé sur le bras long du chromosome $8{ }^{[3]}$ et son séquençage a permis de mettre en évidence plusieurs mutations non-sens et défauts d'épissage responsables de la variabilité des signes cliniques observés dans une fratrie ou entre des patients ayant une origine géographique différente ${ }^{[3,53,55,58] \text {. }}$

\subsection{Histopathologie}

Dans les formes sévères, l'examen radiologique permet le plus souvent de poser le diagnostic d'ostéopétrose ${ }^{[24]}$. En revanche, dans les formes intermédiaires et frustres, les signes radiologiques sont parfois insuffisants. Par contre, l'examen histopathologique met toujours en évidence les altérations pathognomoniques d'un trouble de la résorption osseuse. La biopsie vise à préciser (i) la structure histologique de l'os, (ii) la quantification du nombre d'ostéoclastes présents sur la surface osseuse, (iii) la présence d'une zone claire et d'une bordure en brosse, et (iv) le contenu des cavités médul-

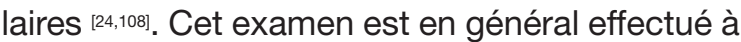
partir d'un prélèvement de crête iliaque réalisé soit à ciel ouvert par l'intermédiaire d'une petite incision cutanée, soit par ponction percutanée à l'aide d'une aiguille de Bordier. Un nombre de plaquettes inférieur à $50000 / \mathrm{mm}^{3}$ constitue une contre-indication à la réalisation de cette biopsie [25].

Les anomalies osseuses reflètent fidèlement les mécanismes pathogéniques responsables de l'ostéopétrose : il y a une forte diminution de la résorption de l'os et du cartilage formés par ossification enchondrale, tandis que leur synthèse est peu ou pas altérée. Ce défaut de résorption entraîne une accumulation progressive de cartilage calcifié dans les métaphyses et les cavités médullaires des diaphyses, ou sous forme d'îlots circulaires entourés d'os fibreux ${ }^{[29,49,79]}$. Dans les formes mineures, par exemple dans la forme bénigne de type I, ils peuvent disparaître spontanément au cours de l'évolution ${ }^{[25,26,39]}$. Dans les formes sévères, le tissu chondro-ostéoïde non résorbé comble progressivement les cavités médullaires, étouffant la moelle hématopoïétique qui se trouve réduite à l'état de vestiges [24]. Le trouble de la résorption osseuse se manifeste également à la périphérie des zones métaphyso-diaphysaires où il entraîne un élargissement et une déformation des métaphyses qui prennent un aspect en forme de massue ${ }^{[49,82] . ~ D a n s ~ l e s ~ d i a p h y s e s, ~ c e ~ t r o u b l e ~ e m p e ̂ c h e ~}$ le remodelage et la tunnellisation de l'os, ce qui aboutit à l'épaississement des corticales. Le trouble du remodelage osseux conduit à une désorientation des fibres collagènes qui ne s'adaptent plus aux forces qu'elles subissent, ce qui entraîne une diminution des propriétés mécaniques de l'os [24,29,32]. La matrice osseuse est extrêmement pauvre en fibrilles collagènes et elles relient rarement les ostéons entre eux.

Les ostéoblastes et les ostéoclastes sont fréquemment groupés en amas. Le nombre de ces derniers peut être augmenté, normal ou diminué selon la forme clinique observée. Une diminution du nombre des ostéoclastes semble refléter une anomalie du développement, tandis qu'une augmentation de leur nombre semble traduire une hyperplasie compensatoire secondaire au ralentissement du turn-over osseux [3]. La taille des ostéoclastes est extrêmement variable, aussi bien chez l'homme que chez les animaux étudiés. La présence d'une bordure en brosse qui caractérise les ostéoclastes actifs, est absente dans plusieurs modèles animaux d'ostéopétrose. Cette absence pourrait traduire un défaut intrinsèque de l'activité ostéoclastique ou un défaut de stimulation de ces derniers par le micro-environnement [3,125-127].

Dans la forme maligne, on retrouve en général un nombre important d'ostéoclastes à la surface des trabécules osseuses. Leurs noyaux sont nombreux mais leurs membranes ne comportent ni bordure en brosse ni zone claire ${ }^{[49]}$. Et on observe médecine

buccale chirurgie buccale

VOL. $12, \mathrm{~N}^{\circ} 3$ 2006

page 155 
fréquemment une prolifération de tissu fibreux à l'intérieur des cavités médullaires [128]. Dans la forme létale, il n'y a que très peu d'ostéoclastes à la surface osseuse ${ }^{[14]}$. Dans la forme bénigne, soit les ostéoclastes sont peu nombreux et dépourvus de bordure en brosse, soit ils sont plus nombreux et plus volumineux [129]. Chez les patients atteints d'un déficit en AC II, on retrouve les mêmes anomalies histopathologiques osseuses que dans les autres formes cliniques ${ }^{[9]}$.

\section{TRAITEMENT}

Toutes les formes cliniques de l'ostéopétrose ont un dénominateur commun : les ostéoclastes sont incapables de résorber normalement le cartilage calcifié et l'os. Plusieurs essais thérapeutiques ont été effectués pour stimuler la production et l'acti- médecine

buccale

chirurgie

buccale

VOL. $12, \mathrm{~N}^{\circ} 3$ 2006

page 156 vité ostéoclastiques in situ ou pour apporter des ostéoclastes par le biais d'une transplantation de moelle osseuse lorsque leur fonction reste déficiente. Les nombreux éléments intervenant dans l'ostéopétrose peuvent potentiellement influencer la réponse au traitement. II doit donc être précédé par un bilan physique, radiologique, biologique et génétique soigneux du patient et de sa famille, et par une anamnèse détaillée de l'évolution de la maladie afin de bien préciser la forme dont le sujet est atteint.

\subsection{Formes communes}

Le traitement des formes communes comporte trois grandes mesures thérapeutiques: la transplantation de moelle osseuse, les traitements hormonaux et diététiques et les traitements symptomatiques.

En 1972, Walker traite des souris ostéopétrotiques par mise en parabiose avec des souris saines [101,102]. A partir de 1977, plusieurs cas d'ostéopétrose maligne humaine ont été sensiblement améliorés par une transplantation de moelle osseuse HLA-compatibles [24,103-105,130-132]. Pour de nombreux patients, il y a une amélioration significative des paramètres cliniques, biologiques, radiologiques et histopathologiques ; les troubles neurologiques peuvent néanmoins persister en dépit d'une transplantation réussie ${ }^{5,25,48,105]}$.

Ce type de traitement ne s'adresse pas à toutes les formes d'ostéopétrose, mais principalement aux formes malignes infantiles. Lorsque les cavités médullaires sont comblées par du tissu chondro-ostéoïde, le succès de la transplantation est compromis ${ }^{[24]}$. Un examen histopathologique méticuleux permet d'évaluer l'efficacité du traitement. Le pronostic à long terme est amélioré par la précocité du traitement qui doit être réalisé si possible avant l'âge de 4 ans [24,132]. Au regard de la multitude d'altérations génétiques et cellulaires potentiellement impliquées, et qui ne sont pas toutes imputables à la seule moelle osseuse, la transplantation de moelle osseuse ne permet pas de traiter toutes les formes malignes $[25,108,130]$. Des transplantations de moelle osseuse non-HLAidentiques ont également été réalisées de manière expérimentale, mais leur efficacité à long terme n'a pas été réellement évaluée ${ }^{[24]}$. Plus récemment, la transplantation de cellules souches sanguines isolées chez des parents HLA-compatibles s'est également révélée efficace ${ }^{[24]}$. La transplantation de moelle osseuse peut toutefois s'accompagner de nombreux effets secondaires, en particulier d'une hypercalcémie secondaire à la reprise brutale et massive de la résorption osseuse [24,25,82].

Les premiers succès dans le traitement de l'ostéopétrose par un régime pauvre en calcium ont été rapportés en 1965 [133]. Des travaux récents montrent qu'une augmentation de l'apport de calcium peut se révéler nécessaire chez les enfants sévèrement atteints, de façon à corriger l'hypocalcémie et le rachitisme induit, secondaires au régime pauvre en calcium et au traitement corticoïde souvent prescrit initialement [134]. Le développement d'un rachitisme peut retarder ou compromettre la transplantation de moelle osseuse et, surtout, en créant une diminution de la densité osseuse au voisinage des cartilages de croissance, simuler une amélioration attribuée à l'efficacité au traitement [34,37]. Le traitement du rachitisme associé à l'ostéopétrose comprend (i) un apport adéquat de calcitriol, (ii) une libéralisation, plutôt qu'une restriction, des apports alimentaires en calcium et (iii) l'administration obligatoire d'une supplémentation calcique lorsqu'un traitement corticoïde est associé $[3,134]$. La vitamine $1,25(\mathrm{OH})_{2} \mathrm{D}_{3}$ (ou calcitriol) qui représente la forme biologique la plus active de la vita- 
mine $D_{3}$, est nécessaire pour la formation et la stimulation des ostéoclastes ${ }^{[135]}$. L'administration de hautes doses de calcitriol, associée à un régime pauvre en calcium destiné à limiter l'hypercalcémie et l'hypercalciurie, a permis dans quelques cas d'obtenir une amélioration clinique très nette, comparable à celle obtenue avec une transplantation de moelle osseuse, probablement par stimulation des ostéoclastes « dormants » [3]. Une résistance à ce traitement peut néanmoins se développer chez les patients traités [106,109]. De même, l'administration intraveineuse au long cours de PTH s'est révélée bénéfique, probablement en favorisant une augmentation de la synthèse rénale de $1,25(\mathrm{OH})_{2} \mathrm{D}_{3}{ }^{[112]}$.

Plus récemment, plusieurs études ont mis une évidence une diminution de la production de superoxydes leucocytaires dans les ostéopétroses sévères. Dans ces cas, l'IFN- $\chi 1 b$ recombinant a permis d'obtenir une évolution favorable sur le plan clinique, biologique et histopathologique $[106,109]$.

Les anomalies hématologiques rencontrées dans l'ostéopétrose maligne résultent principalement de la myélophtysie. Les autres facteurs susceptibles d'aggraver l'anémie sont l'hypersplénisme secondaire à l'hématopoïèse extra-médullaire et probablement l'existence d'autres foyers d'hémolyse qui n'ont pas été encore bien identifiés ${ }^{[3]}$. L'administration de glucocorticoïdes à fortes doses permet d'obtenir une stabilisation du tableau hématologique dans l'ostéopétrose maligne avec pancytopénie et hépatosplénomégalie [34,136]. Un traitement associant la prednisone à une diète pauvre en calcium et riche en phosphate peut être suffisamment efficace pour constituer une alternative à la transplantation de moelle osseuse ${ }^{[34}$. Une décompression chirurgicale des nerfs optique et facial peut se révéler nécessaire ${ }^{[28,74]}$. Pour le nerf optique, les meilleurs résultats sont obtenus avant l'apparition des premiers signes de dégénérescence rétinienne qui doivent être recherchés régulièrement par un examen ophtalmoscopique et électrorétinographique soigneux [27]. Les atteintes visuelles persistent fréquemment après une transplantation de moelle osseuse, il est conseillé de réaliser cette dernière de manière précoce, si possible dès l'âge de 3 mois ${ }^{[3]}$.

\subsection{Déficit en AC II}

II n'y a pas de traitement codifié pour l'ostéopétrose due à un déficit en AC II. Certaines formes d'acidose tubulaire rénale, également associées à des troubles de la croissance, répondent à l'administration de bicarbonate, mais le traitement de l'acidose métabolique associée au déficit en AC II reste un sujet de controverses. L'impact de l'administration au long cours d'une substance alcaline sur le métabolisme demeure inconnu, et il est possible qu'un tel traitement puisse potentiellement réduire l'effet bénéfique de l'acidose métabolique sur la libération de bicarbonates à partir du squelette ${ }^{[0,24]}$. On constate en effet, chez les sujets non traités, une tendance spontanée à la réduction de la densité osseuse au cours de l'évolution ${ }^{[0,24]}$.

La transfusion de globules rouges, ayant un contenu normal en AC II, n'améliore pas l'acidose métabolique. Ceci suggère que l'origine de cette dernière est essentiellement rénale et qu'une éventuelle transplantation de moelle osseuse, pour améliorer le métabolisme squelettique, n'aura aucune influence sur l'acidose tubulaire

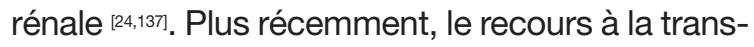
plantation de moelle osseuse, en tant que traitement de base d'un déficit en AC II, a permis une correction du phénotype ostéopétrotique, un ralentissement du développement des calcifications cérébrales, sans toutefois influencer l'acidose tubulaire rénale ${ }^{[24]}$. La vitamine $1,25(\mathrm{OH})_{2} \mathrm{D}_{3}$ s'est révélée être un puissant stimulateur de la transcription du gène de l'AC II. Une association de cette dernière à une diète pauvre en calcium pourrait par conséquent se révéler être d'une très grande utilité dans le déficit en AC II, comme cela a déjà pu être démontré dans d'autres formes d'ostéopétrose [3]. La thérapie génique constituera sans doute l'approche thérapeutique de choix du déficit en AC II. médecine

buccale

chirurgie

buccale

VOL. $12, \mathrm{~N}^{\circ} 3$ 2006

page 157 


\section{RÉFÉRENCES}

117 - TASHIAn RE, Hewett-Emmett D, Dodgson SJ, Forster RE, SLY WS. The value of inherited deficiencies of human carbonic anhydrase isozymes in understanding their cellular roles. Ann N Y Acad Sci 1984 ; 429 : 262-75.

118 - GAY CV, MuelleR WJ. Carbonic anhydrase and osteoclasts: localization by labeled inhibitor autoradiography. Science 1974 ; 183 : 432-4.

119 - VAANANEN HK. Immunohistochemical localization of carbonic anhydrase isoenzymes I and II in human bone, cartilage and giant cell tumor. Histochemistry $1984 ; 81: 485-7$.

120 - Raisz LG, Simmons HA, Thompson WJ, Shepard KL, ANDERSON PS, RODAN GA. Effects of a potent carbonic anhydrase inhibitor on bone resorption in organ culture. Endocrinology 1988 ; 122 : 1083-6.

121 - Blair HC, Teitelbaum SL, Ghiselli R, Gluck S. Osteoclastic bone resorption by a polarized vacuolar proton pump. Science $1989 ; 245$ : 855-7.

122 - LeEs MB, Sapirstein VS, Reiss DS, Kolodny EH. Carbonic anhydrase and 2',3' cyclic nucleotide 3'phosphohydrolase activity in normal human brain and in demyelinating diseases. Neurology 1980 ; $30: 719-25$.

123 - ANDERSON RE, JeE WS, WOOdBURy DM. StimULATION OF CARBONIC ANHYDRASE IN OSTEOCLASTS BY Parathyroid hormone. Calcif Tissue Int 1985 ; 37 : 646-50.

124 - HALL GE, KENNY AD. Bone resorption induced by parathyroid hormone and dibutyryl cyclic AMP: role of carbonic anhydrase. J Pharmacol Exp Ther 1986 ; 238 : 778-82.

125 - Felix R, Cecchini MG, Hofstetter W, Elford PR, StUtZer A, Fleisch H. Impairment of macrophage colony-stimulating factor production and lack of resident bone marrow macrophages in the osteopetrotic op/op mouse. J Bone Miner Res 1990 ; 5 : 781-9.

126 - MARKS SC JR, MACKay CA, SEIFERT MF. The osteopetrotic rabbit: skeletal cytology and ultrastructure. Am J Anat $1987 ; 178: 300-7$.

127 - MARKS SC JR, POPOFF SN. Osteoclast biology in the osteopetrotic (op) rat. Am J Anat 1989 ; 186 : 325-34.
128 - Helfrich MH, ARONSON DC, Everts V, MieREMet RM, GerRistsen EJ, ECKHARDt PG, GROOt CG, Scherft JP. Morphologic features of bone in human osteopetrosis. Bone 1991; 12 : 411-9.

129 - Bollerslev J, Steiniche T, Melsen F, Mosekilde L. Structural and histomorphometric studies of iliac crest trabecular and cortical bone in autosomal dominant osteopetrosis: a study of two radiological types. Bone $1989 ; 10: 19-24$.

130 - Ballet JJ, Griscelli C, Coutris C, Milhaud G, MAROTEAUX P. Bone-marrow transplantation in osteopetrosis. Lancet $1977 ; 2: 1137$.

131 - Sorell M, KapoOR N, KIRKPATRICK D, ROSEN JF, Chaganti RSK, Lopez C, Dupont B, Pollack MS, Terrin BN, Harris MB, Vine D, Rose JS, Goossen C, Good RA, O'REILly RJ. Marrow transplantation for juvenile osteopetrosis. Am J Med 1981; $70: 1280-7$.

132 - Cournot G, Thil CL, Fischer A, Garabédian M. Osteopetrosis: The role of transplantation in marble bone disease. Bone $1989 ; 6: 15-8$.

133 - Dent CE, SMellie JM, WATSOn L. Studies in osteopetrosis. Arch Dis Child $1965 ; 40$ : 7-15.

134 - Kaplan FS, August CS, Fallon MD, Gannon F, HADDAD JG. Osteopetrorickets. The paradox of plenty. Pathophysiology and treatment. Clin Orthop 1993 ; 64-78.

135 - MCSheEHy PM, ChAMBERS TJ. 1,25-dihydroxyvitamin D3 stimulates rat osteoblastic cells to release a soluble factor that increases osteoclastic bone resorption. $\mathrm{J}$ Clin Invest 1987 ; $80:$ 425-9.

136 - OzsoYLu S. High dose intravenous methylprednisolone in treatment of recessive osteopetrosis. Arch Dis Child $1987 ; 62: 214-5$.

137 - WHYTE MP, HAMM LL, SLY WS. Transfusion of carbonic anhydrase-replete erythrocytes fails to correct the acidification defect in the syndrome of osteopetrosis, renal tubular acidosis, and cerebral calcification (carbonic anhydrase-II deficiency). J Bone Miner Res $1988 ; 3: 385-8$. 